\title{
A framework for assessing the relative efficiency of stimulus sequences in evoked response measurements
}

\author{
Barry D. Van Veen \\ Department of Electrical and Computer Engineering, University of Wisconsin-Madison, 1415 Johnson Drive \\ Madison, Wisconsin 53706 \\ Robert E. Lasky \\ Department of Neurology, University of Wisconsin-Madison Medical School, H6/573 CSC, 600 Highland \\ Avenue, Madison, Wisconsin 53792
}

(Received 1 November 1993; accepted for publication 29 June 1994)

\begin{abstract}
A general matrix-based framework for characterizing the performance of cross-correlation techniques for recovering the response to an arbitrary stimulus sequence is presented. In general an infinite number of recovery sequences can be identified for any given stimulus sequence. The recovery sequence that minimizes the noise in the recovered response is derived and the effect of the recovery operation on the noise is analyzed. This general framework is employed to develop analytic expressions for the relative efficiencies of conventional signal averaging and binary MLS based methods for the most commonly used recovery operation and the one that optimizes SNR assuming the background noise is uncorrelated. The results depend on the ratio of response length to MLS minimum pulse interval and the amplitude loss as a function of stimulus rate. Examples based on measured amplitude loss functions for ABR recordings are employed to evaluate the relative efficiencies of MLS techniques. When the noise is uncorrelated conventional signal averaging is from two to five times as efficient as MLS techniques. The relative advantage of averaging is shown to decrease when the noise is dominated by low-frequency components.
\end{abstract}

PACS numbers: 43.64.Ri, 43.64.Yp

\section{LIST OF SYMBOLS}

Lower and upper case boldface symbols are used to represent vector and matrix quantities, respectively. Superscript $T$ denotes matrix transpose and ${ }^{-1}$ denotes matrix inverse. The symbol $\operatorname{tr}(\mathbf{X})$ denotes the trace of the matrix $\mathbf{X}$.

h length $M$ single-pulse stimulus response in the absence of noise

$\mathbf{y}_{i} \quad$ length $N$ measured response to $i$ th presentation of stimulus sequence

$\mathbf{n}_{i} \quad$ length $N$ measurement noise in $i$ th response

A $\quad N$ by $M$ stimulus matrix $k(r) \quad$ dependence of the response amplitude on the minimum pulse interval $r$

y length $N$ averaged response

n length $N$ averaged measurement noise

S $\quad N$ by $N$ measurement noise covariance matrix

$\hat{\mathbf{h}} \quad$ estimated length $M$ single-pulse stimulus response

R $\quad M$ by $N$ recovery matrix

$\mathbf{S}_{h} \quad M$ by $M$ covariance matrix of the noise in $\hat{\mathbf{h}}$

$\sigma^{2} \quad$ total noise variance in $\hat{\mathbf{h}}$

$\alpha \quad$ noise variance in white-noise model for $\mathbf{S}$

$K \quad$ total data collection time

$L \quad$ length of pseudorandom binary sequence

$r \quad$ minimum pulse interval

\section{INTRODUCTION}

The electrophysiologic response to sensory stimulation has been recorded from the surface of the scalp for more than 60 years (Berger, 1929). It is necessary to signal average evoked responses since their amplitudes are small relative to the ongoing electrophysiological activity of the brain (Dawson, 1951). Commercially available evoked response instruments present stimuli separated by fixed interstimulus time intervals (ISls). The lower limit of these fixed intervals is determined by the duration of the electrophysiologic response. Stimulation prior to response completion results in overlapped waveforms which are difficult to interpret. The limit on minimum ISI prohibits more rapid stimulus presen-

tation rates. Faster stimulus rates mean shorter test times which are a high priority for clinical applications. To solve this dilemma, Eysholdt and Schreiner (1982) introduced the use of maximum length sequence (MLS) stimuli and crosscorrelation techniques to recover auditory brain-stem evoked responses (ABRs). A number of investigators have now demonstrated that it is possible to obtain reliable ABRs using MLS techniques at rates up to 1000 clicks/s (Eysholdt and Schreiner, 1982; Burkard et al., 1990a, b; Burkard, 1991; Chan et al., 1992; Lasky et al., 1992; Marsh, 1992; Picton et al., 1992).

The faster stimulation rates possible with crosscorrelation techniques do not necessarily imply faster data 
collection for a variety of reasons. Prominent among these are:

(1) Response amplitude is a decreasing function of rate at both conventional rates and the faster rates possible with MLS stimulation (Lasky and Rupert, 1982; Burkard, 1991; Lasky et al., 1992). Thus, for a constant number of stimuli, signal-to-noise ratio (SNR) decreases with increasing stimulus rate.

(2) Signal averaging is more efficient than MLS techniques at improving the SNR of evoked responses in the presence of uncorrelated noise (Burkard, 1991; Marsh, 1992). Therefore, a greater number of MLS stimuli must be presented to attain the same SNR. Note that signal averaging is necessarily more efficient only if the noise is uncorrelated, that is, spectrally white.

(3) Noisy subjects (i.e., subjects with high levels of surface recorded electrical activity unrelated to the neural response to the stimulation) have reduced SNRs. Consequently, rejecting electrical activity which exceeds reasonable levels (i.e., artifact rejection) has been widely employed to improve SNRs. Rejection of a transient artifact only removes the response to one stimulus when signal averaging but removes all the responses to a MLS stimulus unless additional assumptions are made.

The primary contribution of this paper is development of a general linear algebraic framework for analysis and improvement of cross-correlational techniques. This framework characterizes the recovery of responses of interest and the effects of the recovery operation on the noise. It is applicable to arbitrary stimulus sequences, e.g., binary, multilevel, and white noise. This framework directly leads to the following contributions:

(1) We develop a straightforward approach for addressing the issue of relative time savings afforded by crosscorrelation techniques. The relative time savings depends on the noise covariance sequence and the recovery method.

(2) We show that an infinite number of recovery sequences generally can be identified for any given stimulus sequence. Consequently, the recovery sequences that are most appropriate in a given situation depend on a knowledge of the properties of the sequences, the noise characteristics of the subject, and the purpose of the investigation.

(3) Recovery sequences that minimize the noise in the recovered response are derived. They should be selected if the motivation is to improve the SNR of the recorded data in order to minimize data collection time. To our knowledge this has not been done.

(4) We show that in general the recovery process modifies the noise characteristics. If the measurement noise is white, the noise in the recovered response is usually colored. Furthermore, the variance of the noise can be different at different times in the recovered response. The recovered response noise covariance matrix is shown to depend on the covariance of the measurement noise and the recovery sequence. Knowledge of the recovered response noise characteristics is important for correct interpretation of the response. For example, implementation of a hypothesis test to ascertain the presence or absence of a response requires knowledge of the noise covariance.
(5) We employ the general framework to compare and contrast the relative noise performance of binary MLS based methods and conventional signal averaging when the noise is uncorrelated. Two different recovery sequences are analyzed in the MLS case: one that is commonly utilized (Burkard et al., 1990a, b; Burkard, 1991; Lasky et al., 1992, 1993) and another that optimizes the SNR. Analytic expressions are developed for the relative efficiencies of the three approaches that depend on the ratio of the response length (which defines the fastest conventional signal averaged rate possible) to MLS minimum pulse interval (MPI) and the amplitude loss as a function of rate.

(6) We also compare and contrast the relative noise performance of three binary MLS based methods and conventional signal averaging for colored noise having a low-pass characteristic typical of biologic noise.

Section I develops the general framework using a matrix-based model for the measured response. The recovery process and its relationship to SNR are addressed in Sec. II. Section III compares binary MLS and conventional signalaveraging methods and includes three examples based on the ABR. The ABR examples are selected because most research interest in MLS techniques in the auditory system has focused on ABRs. This interest is due to Eysholdt and Schreiner's (1982) work and the clinical importance of ABR recordings. Section IV concludes the paper with a discussion.

\section{MATRIX BASED RESPONSE MODEL}

The representation derived in this section assumes that a known stimulus sequence is repetitively presented to a subject and that the response to a sequence of stimuli is the sum of the responses to individual stimuli. We also assume that the response amplitude is stimulus dependent. This models the reduction in response amplitude that occurs with increasing presentation rate of pulse stimuli (Lasky and Rupert, 1982; Burkard, 1991; Lasky et al., 1992). All of these assumptions can be relaxed somewhat at the expense of complicating the analysis. Our analysis makes extensive use of linear algebra. A good reference on linear algebra is Noble and Daniel (1977).

Let the samples of the response to a unit amplitude pulse be denoted by the $M$ by one vector $\mathbf{h}$ and let the $N$ by one $(N \geqslant M)$ vector $\mathrm{y}_{i}, i=1,2, \ldots, I$, represent the response to the $i$ th presentation of the stimulus sequence. Hence, the stimulus sequence has period $N$ and is presented to the subject $I$ times. The response $y_{i}$ consists of a sum of scaled and shifted versions of the unit pulse response $h$ resulting from the convolution of $\mathbf{h}$ with the stimulus sequence. Noise, represented by the $N$ by one vector $\mathbf{n}_{i}$, is also present. Hence, in matrix notation

$$
\mathbf{y}_{i}=\mathbf{A h} k(r)+\mathbf{n}_{i}, \quad i=1,2, \ldots, I .
$$

The $N$ by $M$ stimulus matrix $\mathbf{A}$ represents the scaling and shifting of the response associated with the particular stimulus sequence, that is, the product $A h$ is the convolution of the stimulus sequence with $\mathbf{h}$. The scale factor $k(r)$ describes the response amplitude dependence on the stimulus sequence.

The stimulus matrix $\mathbf{A}$ is constructed as follows. The first column of $\mathbf{A}$ is given by the length $N$ stimulus sequence. 
The $k$ th column, $k=2,3, \ldots, M$ is obtained by circularly shifting the first column $k-1$ times. The circular shift is necessary because the stimulus sequence is presented repetitively and accounts for the overlap between the responses elicited by the previous period of the stimulus sequence and the present one. Repetitive stimulus presentation induces a circular convolution operation. If the stimulus sequence is not presented repetitively, then linear shifts are used to construct the second through $M$ th columns of $\mathbf{A}$ and $\mathbf{A}$ contains all zeros above the main diagonal.

An example serves to illustrate the nature of the stimulus matrix construction. Suppose the stimulus sequence is given

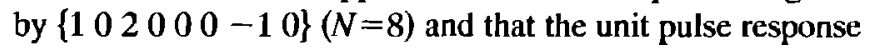
$h$ is length $M=4$. It is straightforward to show that

$$
\mathbf{A}=\left[\begin{array}{cccc}
1 & 0 & -1 & 0 \\
0 & 1 & 0 & -1 \\
2 & 0 & 1 & 0 \\
0 & 2 & 0 & 1 \\
0 & 0 & 2 & 0 \\
0 & 0 & 0 & 2 \\
-1 & 0 & 0 & 0 \\
0 & -1 & 0 & 0
\end{array}\right] .
$$

If conventional signal averaging is used with an ISI of $M$, then $\mathrm{A}$ is the $M$ by $M$ identity matrix.

The responses to each presentation of the stimulus sequence are usually averaged to improve the signal-to-noise ratio. Let the averaged response $\mathbf{y}$ be given by

$$
\mathrm{y}=\frac{1}{I} \sum_{i=1}^{I} \mathrm{y}_{i} .
$$

Substitution of the model (1) yields

$$
\mathbf{y}=\operatorname{Ah} k(r)+\mathbf{n},
$$

where $n=1 / I \sum_{i=1}^{I} n_{i}$. Assume the noise vectors $n_{i}$, $i=1,2, \ldots, I$ are zero mean and identically distributed with covariance matrix $\operatorname{Cov}\left(\mathbf{n}_{i}\right)=E\left\{\mathbf{n}_{i} \mathbf{n}_{i}^{T}\right\}=\mathbf{S}$. If the noise vectors are uncorrelated, that is, $E\left\{\mathbf{n}_{i} \mathbf{n}_{j}^{T}\right\}=\mathbf{0}$ for $i \neq j$, then averaging reduces the noise variance in each element of $\mathbf{y}$ by a factor of I. Equivalently, we have $\operatorname{Cov}(\mathbf{n})=1 / I S$.

\section{THE RECOVERY PROCESS AND SIGNAL-TO- NOISE RATIO}

The goal is usually to obtain the best possible estimate of the unit pulse response $h$. The measured response $y$ contains a sum of scaled and shifted versions of $h$ plus noise so an additional sequence of operations is necessary to "recover" $h$ from $y$. These operations are linear and thus are represented by multiplying y with an $M$ by $N$ recovery matrix R. This general perspective includes previously reported recovery methods (Eysholdt and Schreiner, 1982; Burkard et al., 1990a, b; Burkard, 1991; Chan et al., 1992; Lasky et al., 1992; Marsh, 1992; Picton et al., 1992).

Let $\hat{\mathbf{h}}=\mathbf{R y}$ represent the estimate of $\mathbf{h}$. Substitute (4) for $\mathrm{y}$ and temporarily assume $k(r)$ is unity to obtain

$$
\hat{\mathbf{h}}=\mathbf{R A h}+\mathbf{R n} \text {. }
$$

Since the noise $\mathbf{n}$ is zero mean, an unbiased estimate of $\mathbf{h}$ is obtained provided

$$
\mathbf{R A}=\mathbf{I} \text {, }
$$

where $I$ is the $M$ by $M$ identity matrix. Condition (6) can always be satisfied if and only if $A$ is a rank $M$ matrix. This implies that the stimulus sequence and its first $M-1$ circular shifts must be linearly independent in order to recover the unit pulse response. Now, if $N>M$ and $\mathbf{A}$ is rank $M$, then there are an infinite number of matrices $\mathbf{R}$ that satisfy (6). Given any solution to (6) one can obtain another solution by adding a component to any row of $\mathbf{R}$ that lies in the space orthogonal to the space spanned by the columns of $\mathbf{A}$.

In cases where an infinite number of recovery matrices exist that satisfy (6), choice of a unique recovery matrix must be decided by other criteria. Computational complexity considerations suggest choosing an $\mathbf{R}$ that is relatively sparse or contains particularly simple entries, such as \pm 1 . Unfortunately, systematic means for determining such $\mathbf{R}$ do not exist. Furthermore, computational complexity is often not a primary concern since computation of $\mathbf{R y}$ for general $\mathbf{R}$ is well within the capability of current generation computing hardware, except perhaps for extremely long stimulus sequences.

An alternate criterion is based on minimizing the contribution of the noise to the estimate $\hat{\mathbf{h}}$. Let $\mathbf{S}_{h}=\operatorname{Cov}(\hat{\mathbf{h}})$. The diagonal elements of $S_{h}$ represent the noise variance in the corresponding elements of the estimate $\hat{\mathbf{h}}$. Note that

$$
\mathbf{S}_{h}=\operatorname{Cov}(\mathbf{R n})=\mathbf{R} \operatorname{Cov}(\mathbf{n}) \mathbf{R}^{T}=1 / I \mathbf{R S R}^{T},
$$

so clearly the noise performance depends on both the noise in the measured response $y$ and the recovery process. In general the diagonal elements of $S_{h}$ are not equal, that is, the noise variance in differing elements of the estimate $\hat{h}$ are different. In order to obtain a global measure of noise performance, we adopt the total noise variance

$$
\sigma^{2}=\operatorname{tr}\left(S_{h}\right) \text {. }
$$

Consider choosing $\mathbf{R}$ to minimize the total noise variance (8) subject to the constraint (6), that is,

$$
\min _{\mathbf{R}} \operatorname{tr}\left(\mathbf{R S R}^{T}\right) \text { subject to } \mathbf{R A}=\mathbf{I} \text {. }
$$

The solution to (9) is derived in the Appendix and is given by

$$
\mathbf{R}=\left(\mathbf{A}^{T} \mathbf{S}^{-1} \mathbf{A}\right)^{-1} \mathbf{A}^{T} \mathbf{S}^{-1} .
$$

This $\mathbf{R}$ results in an estimate of $\mathbf{h}$ known as the best linear unbiased estimator (Kay, 1993). It can be shown that the solution (10) independently minimizes each diagonal element of $S_{h}$ (Kay, 1993). This implies that (10) also minimizes the noise variance at each point in the estimated response $\hat{\mathbf{h}}$. Substitution of (10) into (7) yields

$$
S_{h}=(1 / I)\left(A^{T} S^{-1} A\right){ }^{1} \text {. }
$$

Evaluation of (10) requires knowledge of the noise covariance matrix $\mathbf{S}$. If $\mathbf{S}$ is unknown, the sample covariance 
estimate can be employed either using the measured responses $\mathrm{y}_{i}, i=1,2, \ldots, I$,

$$
\hat{\mathbf{S}}=\frac{1}{I-1} \sum_{i=1}^{I}\left(\mathrm{y}_{i}-\mathbf{y}\right)\left(\mathrm{y}_{i}-\mathbf{y}\right)^{T},
$$

or using $J$ noise only measurements $\mathbf{n}_{j}, j=1,2, \ldots, J$,

$$
\hat{\mathbf{S}}=\frac{1}{J} \sum_{j=1}^{J} \mathbf{n}_{j} \mathbf{n}_{j}^{T} .
$$

Clearly a combination of (12) and (13) can also be used. It can be shown (Muirhead, 1982) that the sample covariance estimate is nonsingular with probability one if the noise has a continuous probability density function and $I>N$ or $J \geqslant N$. As a rule of thumb, $I$ or $J$ should be several times as large as $N$ to obtain a statistically reliable estimate of $\mathbf{S}$. Note that the recovery matrix (10) no longer exactly minimizes the noise variance when the true covariance matrix is replaced with an estimate.

In the special case where the noise is uncorrelated from sample to sample, that is, spectrally white, then $S=\alpha I$ where $\alpha$ is the noise variance and (10) simplifies to

$$
\mathbf{R}=\left(\mathbf{A}^{T} \mathbf{A}\right)^{-1} \mathbf{A}^{T} \text {. }
$$

This recovery matrix corresponds to the minimum norm pseudoinverse of the matrix $\mathbf{A}$. The noise covariance matrix $S_{h}$ is now

$$
\mathbf{S}_{h}=\alpha / I\left(\mathbf{A}^{T} \mathbf{A}\right)^{-1} .
$$

Note that the noise in the estimate $\hat{\mathbf{h}}$ is generally correlated or colored even if the noise in the data is uncorrelated or white. Furthermore, the noise variance can vary from one element of $\hat{\mathbf{h}}$ to another since the diagonal elements of $\left(\mathbf{A}^{T} \mathbf{A}\right)^{-1}$ are not necessarily equal.

A variety of definitions of the signal-to-noise ratio (SNR) are possible although differing definitions will yield slightly different results when evaluating noise performance. We define SNR as the ratio of total energy in the unit pulse response to the total noise variance

$$
\mathrm{SNR}=\frac{k^{2}(r) \mathbf{h}^{T} \mathbf{h}}{\operatorname{tr}\left(\mathrm{S}_{h}\right)}
$$

This is equivalent to the ratio of average energy in the unit pulse response to average noise variance. In the special case of uncorrelated noise $(\mathbf{S}=\alpha \mathbf{I})$,

$$
\mathrm{SNR}=\frac{I k^{2}(r) \mathbf{h}^{T} \mathbf{h}}{\alpha \operatorname{tr}\left(\mathbf{R R}^{T}\right)} .
$$

Furthermore, if $\mathbf{R}$ is given by (14),

$$
\mathrm{SNR}=\frac{I k^{2}(r) \mathbf{h}^{T} \mathbf{h}}{\alpha \operatorname{tr}\left[\left(\mathbf{A}^{T} \mathbf{A}\right)^{-1}\right]} .
$$

An example serves to illustrate the main points of this section. Consider the binary stimulus sequence $\left\{\begin{array}{lllllll}1 & 1 & 0 & 1 & 0 & 0 & 1\end{array}\right\}$ with a minimum interstimulus interval of two samples and response length $M=5$. Hence $N=14$ and

$$
\mathbf{A}=\left[\begin{array}{lllll}
1 & 0 & 1 & 0 & 0 \\
0 & 1 & 0 & 1 & 0 \\
1 & 0 & 1 & 0 & 1 \\
0 & 1 & 0 & 1 & 0 \\
0 & 0 & 1 & 0 & 1 \\
0 & 0 & 0 & 1 & 0 \\
1 & 0 & 0 & 0 & 1 \\
0 & 1 & 0 & 0 & 0 \\
0 & 0 & 1 & 0 & 0 \\
0 & 0 & 0 & 1 & 0 \\
0 & 0 & 0 & 0 & 1 \\
0 & 0 & 0 & 0 & 0 \\
1 & 0 & 0 & 0 & 0 \\
0 & 1 & 0 & 0 & 0
\end{array}\right] .
$$

It is easy to verify that the recovery matrix $R_{1}$

$$
\mathbf{R}_{1}=\frac{1}{4}\left[\begin{array}{cccccccccccccc}
1 & 0 & 1 & 0 & -1 & 0 & 1 & 0 & -1 & 0 & -1 & 0 & 1 & 0 \\
0 & 1 & 0 & 1 & 0 & -1 & 0 & 1 & 0 & -1 & 0 & -1 & 0 & 1 \\
1 & 0 & 1 & 0 & 1 & 0 & -1 & 0 & 1 & 0 & -1 & 0 & -1 & 0 \\
0 & 1 & 0 & 1 & 0 & 1 & 0 & -1 & 0 & 1 & 0 & -1 & 0 & -1 \\
-1 & 0 & 1 & 0 & 1 & 0 & 1 & 0 & -1 & 0 & 1 & 0 & -1 & 0
\end{array}\right]
$$

satisfies $\mathbf{R}_{\mathbf{1}} \mathbf{A}=\mathbf{I}$. However, although $\mathbf{R}_{1}$ is computationally simple to implement, it does not have optimal noise properties. Assuming $S=\alpha I$, the noise covariance $S_{h}$ associated with (20) is

$$
S_{h}=\frac{\alpha}{16 I}\left[\begin{array}{ccccc}
7 & 0 & -1 & 0 & -1 \\
0 & 7 & 0 & -1 & 0 \\
-1 & 0 & 7 & 0 & -1 \\
0 & -1 & 0 & 7 & 0 \\
-1 & 0 & -1 & 0 & 7
\end{array}\right]
$$

so the total noise power (8) is $\sigma=35 \alpha / 16 I$. In contrast, (14) gives 


$$
\mathbf{R}=\frac{1}{24}\left[\begin{array}{cccccccccccccc}
6 & 0 & 3 & 0 & -6 & 0 & 6 & 0 & -3 & 0 & -3 & 0 & 9 & 0 \\
0 & 4 & 0 & 4 & 0 & -4 & 0 & 8 & 0 & -4 & 0 & 0 & 0 & 8 \\
6 & 0 & 3 & 0 & 6 & 0 & -6 & 0 & 9 & 0 & -3 & 0 & -3 & 0 \\
0 & 4 & 0 & 4 & 0 & 8 & 0 & -4 & 0 & 8 & 0 & 0 & 0 & 4 \\
-6 & 0 & 3 & 0 & 6 & 0 & 6 & 0 & -3 & 0 & 9 & 0 & -3 & 0
\end{array}\right]
$$

Again assuming $\mathbf{S}=\alpha I$, the noise covariance $\mathbf{S}_{h}$ associated with (22) is

$$
\mathbf{S}_{h}=\frac{\alpha}{24 I}\left[\begin{array}{ccccc}
9 & 0 & -3 & 0 & -3 \\
0 & 8 & 0 & -4 & 0 \\
-3 & 0 & 9 & 0 & -3 \\
0 & -4 & 0 & 8 & 0 \\
-3 & 0 & -3 & 0 & 9
\end{array}\right]
$$

resulting in a total noise power (8) of $\sigma=43 \alpha / 24 I$. In this case the noise power obtained using the recovery matrix (20) is a factor of 1.221 larger than that obtained using the optimal recovery matrix (22). Hence, the SNRs also differ by a factor of 1.221. Note also that the noise covariance structure differs so the spectral distribution of the noise in the recovered response is different for these two recovery matrices.

\section{MAXIMUM LENGTH SEQUENCES AND CONVENTIONAL SIGNAL AVERAGING}

The framework derived in the previous sections is now used to compare the relative efficiencies of binary MLS stimuli and conventional signal averaging from an SNR perspective. In general the relative merits of the two approaches are strongly dependent on the noise covariance matrix $\mathbf{S}$. Here the special case of uncorrelated noise $(\mathbf{S}=\alpha \mathbf{I})$ is addressed.

Begin with conventional averaging. Assuming the total data collection time is $K$, the response length is $M$, and the maximum stimulus rate is used (one every $M$ samples), then a total of $K / M$ responses are averaged. Hence, the SNR defined in (16) is

$$
\mathrm{SNR}_{\mathrm{av}}=\frac{K \mathbf{h}^{T} \mathbf{h}}{\alpha M^{2}} .
$$

Here we have assumed that the amplitude function, $k(r)$, is unity.

Now consider the MLS case. The MLS is a pseudorandom binary sequence of length $L$ equal to $2^{d}-1$ where $d$ is the order of the sequence. $(L+1) / 2$ elements in the sequence have a value +1 and the remainder have a value -1 . The autocorrelation function of MLS equals $L$ at a shift of zero and -1 at all other shifts. These sequences can be generated by the algorithms of Davies (1966). We assume that the bipolar MLS is converted to a monopolar sequence by using the values +1 to represent a stimulus and -1 to represent the absence of a stimulus. This type of stimulus is commonly used for recording auditory brain-stem responses (Hall, 1992). The rate of stimulus presentation is controlled by inserting $r-1$ zeros after each entry of the original monopolar sequence. This results in a total sequence length of $N=L$ r with a MPI of $r$ sample units. The stimulus sequence used in the example at the end of the previous section is a length $L=7$, order $d=3$, monopolar MLS with MPI $=r=2$.

The MLS response SNR is evaluated for two different recovery matrices: one constructed from the original bipolar MLS and a second given by the solution to (14). We begin with the former. It is easy to demonstrate that a recovery matrix constructed in the following manner satisfies the condition (6). Set the first row of $\mathbf{R}$ equal to the original bipolar MLS divided by $(L+1) / 2$ with $r-1$ zeros inserted after each entry. The $i$ th, $i=2,3, \ldots, M$, row is set equal to the first row circularly shifted $i$ times. This is the procedure used to generate the recovery matrix of $(20)$. It is straightforward to verify that the $M$ diagonal elements of $\mathbf{R} \mathbf{R}^{T}$ are all equal to $4 L /(L+1)^{2}$. Again assuming a total data collection time of $K$, a total of $K /(L r)$ responses are averaged. Hence the SNR is

$$
\mathrm{SNR}_{\mathrm{MLSI}}=\frac{K(L+1)^{2} k^{2}(r) \mathbf{h}^{T} \mathbf{h}}{4 \alpha L^{2} M r} .
$$

In most applications $L$ is sufficiently large that $(L+1) / L \approx 1$ and thus

$$
\mathrm{SNR}_{\mathrm{MLS} 1} \approx \frac{K k^{2}(r) \mathbf{h}^{T} \mathbf{h}}{4 \alpha M r} .
$$

The case where the recovery matrix is designed accord ing to (14) is slightly more complicated. Here the SNR is given by (18). It is straightforward to verify that the matrix $\mathbf{A}^{T} \mathbf{A}$ is of the form $\mathbf{Q}(L+1) / 4$ where the $M$ by $M$ matrix $\mathbf{Q}$ has two on the main diagonal, one on each $r$ th sub- and superdiagonal, and zero elsewhere. Thus the SNR is

$$
\mathrm{SNR}_{\mathrm{MLS} 2}=\frac{K(L+1) k^{2}(r) \mathbf{h}^{T} \mathbf{h}}{4 \alpha L r \operatorname{tr}\left(\mathbf{Q}^{-1}\right)} .
$$

An expression for $\operatorname{tr}\left(\mathbf{Q}^{-1}\right)$ in terms of $M$ and $r$ is difficult to obtain in general. However, a very simple expression can be obtained for the case where $M / r$ is an integer. This case leads to a conjecture for an expression in the general case.

Recall that the trace of a matrix is equal to the sum of its eigenvalues and that the eigenvalues of $\mathbf{Q}^{-1}$ are the inverse of the eigenvalues of $\mathbf{Q}$. Now $\mathbf{Q}$ is expressed as $\mathbf{I}+\mathbf{C}$ where $\mathrm{C}$ is one on the main diagonal and every $r$ th sub- and superdiagonal. The eigenvalues of $\mathbf{Q}$ are equal to the eigenvalues of $\mathbf{C}$ plus one. If $M / r$ is an integer, then the matrix $\mathbf{C}$ can be expressed as the Kronecker product

$$
\mathbf{C}=\mathbf{1}_{M / r} \otimes \mathbf{I}_{r},
$$




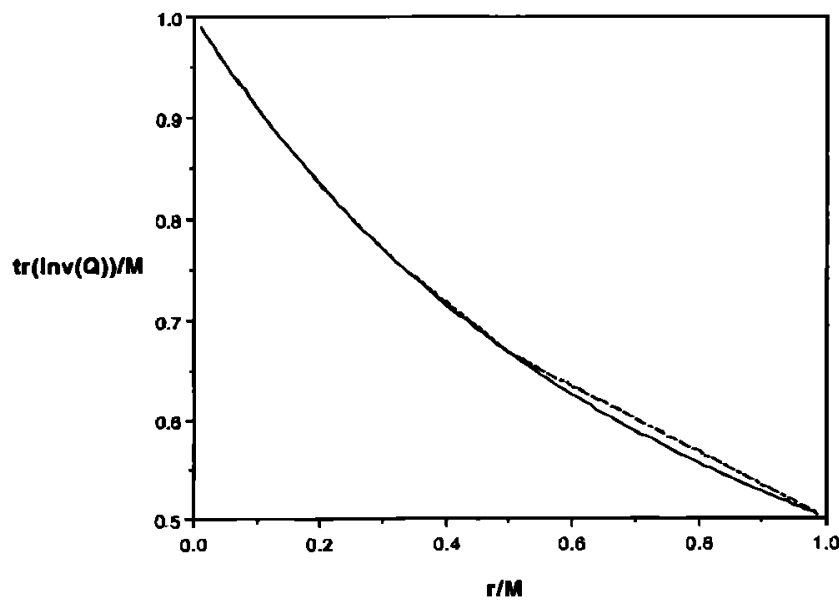

FIG. 1. $M^{1} \operatorname{tr}\left(Q^{-1}\right)$ evaluated exactly (-.-.) and approximated using Eq. (29) (—).

where $\mathbf{I}_{r}$ denotes the $r$ by $r$ identity matrix and $\mathbf{1}_{M / r}$ denotes a $M / r$ by $M / r$ matrix containing ones. The eigenvalues of a Kronecker product of matrices are given by all possible products of the eigenvalues of the constituent matrices (Brewer, 1978). The $r$ eigenvalues of $\mathbf{I}_{r}$ are unity and it is easy to show that $1_{M / r}$ has one eigenvalue equal to $M / r$ and the rest are zero. Hence, $\mathrm{C}$ has $r$ eigenvalues equal to $M / r$ and $M-r$ eigenvalues equal to zero. This implies that $\mathbf{Q}$ has $r$ eigenvalues equal to $M / r+1$ and $M-r$ eigenvalues equal to one so that

$$
\operatorname{tr}\left(\mathbf{Q}^{-1}\right)=r \frac{r}{M+r}+M-r=M\left(\frac{M / r}{1+M / r}\right) .
$$

Empirical evidence suggests that (29) is an excellent approximation to $\operatorname{tr}\left(Q^{1}\right)$ when $M / r$ is not an integer. Figure 1 depicts $M^{1} \operatorname{tr}\left(Q^{-1}\right)$ as a function of $r / M$ evaluated numerically and using (29). There is no perceptible difference between these two functions for $r / M \leqslant 0.5$ and only a slight difference for $r / M>0.5$. Close examination of $M^{-1} \operatorname{tr}\left(\mathbf{Q}^{-1}\right)$ suggests that the value at noninteger $M / r$ is given by linear interpolation of (29) evaluated at the nearest integer values of $M / r$. Substituting (29) into (27) and approximating ( $L$ $+1) / L \approx 1$ yields

$$
\mathrm{SNR}_{\mathrm{MLS} 2} \approx \frac{K(1+M / r) k^{2}(r) \mathbf{h}^{T} \mathbf{h}}{4 \alpha M^{2}} .
$$

Equations (24), (26), and (30) are now used to compare the performance of the three approaches. Consider the ratios of SNRs

$$
\begin{aligned}
\frac{\mathrm{SNR}_{\mathrm{av}}}{\mathrm{SNR}_{\mathrm{MLS} 1}} & =\frac{4}{k^{2}(r)(M / r)}, \\
\frac{\mathrm{SNR}_{\mathrm{av}}}{\mathrm{SNR}_{\mathrm{MLS} 2}} & =\frac{4}{k^{2}(r)(1+M / r)}, \\
\frac{\mathrm{SNR}_{\mathrm{MLS1}}}{\mathrm{SNR}_{\mathrm{MLS} 2}} & =\frac{M / r}{1+M / r} .
\end{aligned}
$$

These results are independent of the MLS sequence length $L$ provided $L$ is sufficiently large [The dependence on $L$ is

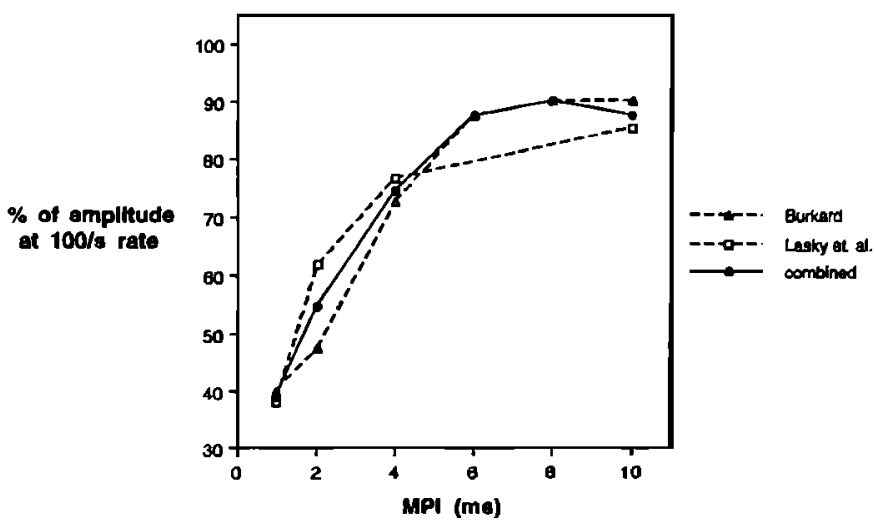

FIG. 2. Wave $V$ amplitude reduction as a function of MLS presentation rates-data from Burkard et al. (1990b), Lasky et al. (1992), and the average (combined) of the two studies.

easily included by comparing (24), (25), and (27)]. As expected, $S_{N R R_{M L S 2}}>S_{N R R_{M L S 1}}$ although the differential is small when $M / r$ is large. The comparison between conventional signal averaging and the MLS stimulus sequence depends on the rate amplitude loss factor $k(r)$. However, even if $k(r)$ is unity, we must have $M / r>4$ and $M / r>3$, respectively, for the MLS1 and MLS2 approaches to improve upon the SNR obtained by conventional signal averaging.

It is straightforward to recast the efficiency question in terms of the relative data collection times $(K)$ required to obtain a given SNR. It can be shown that the ratio of SNRs is the inverse of the ratio of data collection times. Specifically,

$$
\begin{aligned}
& \frac{K_{\mathrm{av}}}{K_{\mathrm{MLS1}}}=\frac{k^{2}(r)(M / r)}{4}, \\
& \frac{K_{\mathrm{av}}}{K_{\mathrm{MLS} 2}}=\frac{k^{2}(r)(1+M / r)}{4}, \\
& \frac{K_{\mathrm{MLS} 1}}{K_{\mathrm{MLS} 2}}=\frac{1+M / r}{M / r} .
\end{aligned}
$$

Example 1: The most frequently proposed application for MLS techniques in the auditory system is the screening of auditory function by recording ABRs. Since waveform morphology deteriorates with increasing rate (Hall, 1992), MLS techniques are likely to be considered when it is sufficient to determine only that the subject responded to the stimulus. Consequently, MLS rates should be compared with the conventional signal averaged rate that achieves a desired Wave V SNR in the shortest time, generally, the fastest conventional rate possible (approximately, 100/s).

Currently, there are two published studies which recorded ABRs to a range of MLS rates and to a conventional stimulus at close to 100/s from human adults (Burkard et al., 1990b; Lasky et al., 1992). Figure 2 plots the reduction in Wave $\mathrm{V}$ amplitude relative to the conventional 100/s Wave $\mathrm{V}$ amplitude as a function of MLS minimum pulse interval reported in each of these studies. In addition, the average of the reductions observed for both studies is also plotted. There is good agreement between the two studies. We use the average of the two studies as the rate amplitude loss factor $k(r)$ : the 


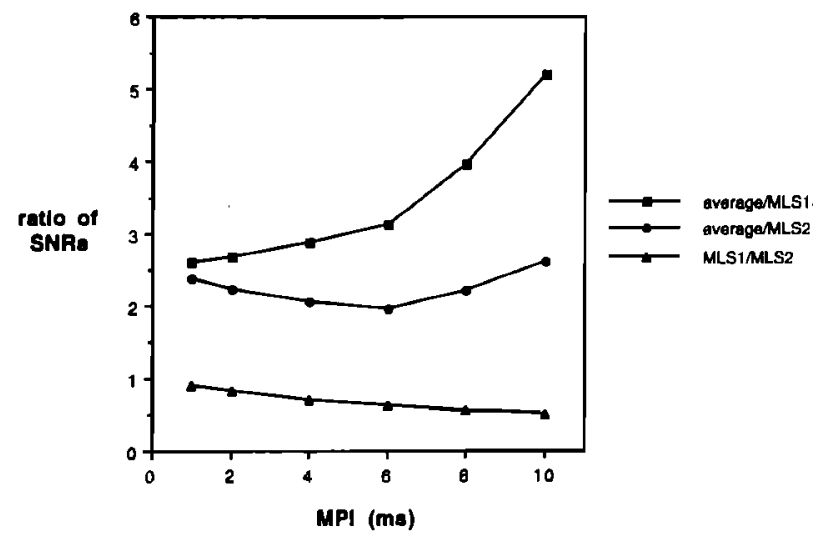

FIG. 3. Ratios of SNRs as a function of MLS presentation rates for conventional signal averaging (average) and two MLS response recovery matrices [MLS1 constructed from the original bipolar MLS and MLS2 minimizing the noise variance, Eq. (14)] assuming white measurement noise.

relative efficiencies of conventional signal averaging and MLS response recovery when the noise variance is minimized and when it is not is evaluated using (31)--(33). The result of this comparison is depicted in Fig. 3.

These results indicate that for equivalent data collection times the SNR is always better with conventional signal averaging than the MLS methods by a factor of $2-5$. Alternatively, for equivalent SNR the data collection time is from two to five times shorter with averaging than with MLS methods. Note that the optimal SNR recovery sequence offers advantages in SNR or data collection time relative to the conventional recovery sequence, especially for moderate MPIs.

Example 2: Here we repeat the previous ABR example assuming the noise is correlated. The noise spectrum is depicted in Fig. 4. This shape was chosen to approximate noise measurements made in our laboratory and reflects the fact that biological noise tends to be largest at low frequencies. The SNR expressions derived in this section for white noise are no longer applicable so we use (16) and (7) to evaluate the ratios of conventional averaging SNR to MLS based SNRs. Three different MLS based recovery matrices are con-

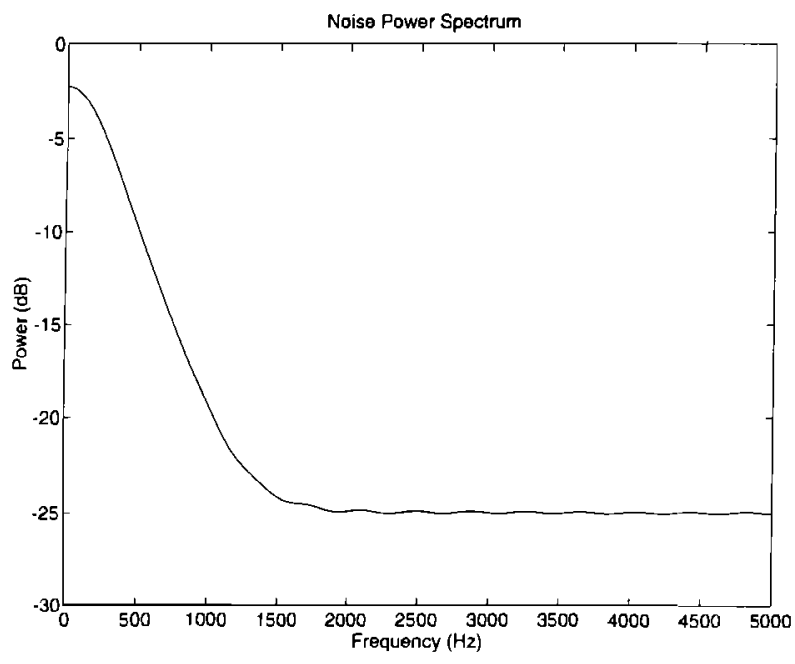

FIG. 4. Power spectrum of the noise used in example: 2.

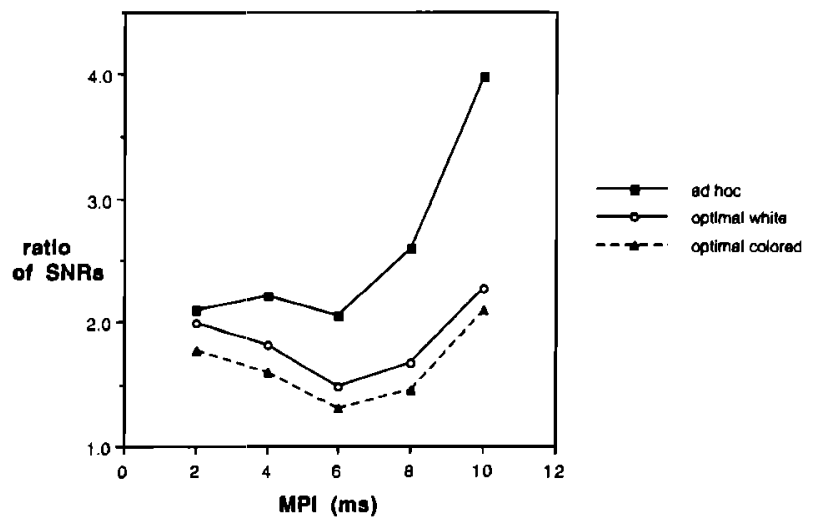

FIG. 5. Ratios of conventional signal averaging SNR to MLS based SNRs as a function of MLS presentation rate assuming the colored measurement noise depicted in Fig. 4. The three MLS approaches are the ad hoc recovery method, optimum recovery method for white noise, and optimum recovery method for colored noise.

sidered: the commonly used ad hoc recovery matrix that was previously denoted as MLS1, the optimum recovery matrix for white noise given by (14), and the optimum recovery matrix for colored noise given by (10). The sampling frequency is $10 \mathrm{kHz}$, response length is $10 \mathrm{~ms}(M=100)$, and the MLS sequence length $L=7$.

The ratios of SNRs depicted in Fig. 5 demonstrate that conventional averaging remains more efficient than the MLS methods although the differential is less than the white-noise case. The optimal colored noise recovery method achieves the best performance by exploiting knowledge of the noise covariance matrix. The optimal white-noise recovery method offers slightly less SNR than the optimal colored noise approach. Both of these methods offer performance advantages over the commonly used ad hoc method. The difference between the optimal white and colored noise approaches is likely to increase if the noise becomes more strongly colored.

Example 3: In this example we evaluate the ABR of a normal human adult using the optimum white noise and commonly used recovery methods to empirically demonstrate that the two methods yield comparable results under low noise conditions. An order 6 MLS having MPI $=2.2 \mathrm{~ms}$ was presented at $90 \mathrm{~dB}$ SPL and the response averaged 200 times. This condition was repeated to obtain two independent responses to the MLS. Note that a short MPI was used to minimize the differences in SNR of the recovered response (see Fig. 5). Furthermore, a large stimulus level was selected to maximize the response level. Figure 6 depicts the ABRs obtained from each trial using the optimum white noise and commonly used ad hoc recovery methods. There are very slight differences between the ABRs computed using the two methods.

This example demonstrates that the recovered responses do not substantially differ as a consequence of applying the two recovery methods when they should be comparable (i.e., short MPI and high SNR). Whether the optimal method affords savings in acquisition time due to an expected improvement in SNR is a question which can only be answered by a large empirical study since the improvement is rela- 

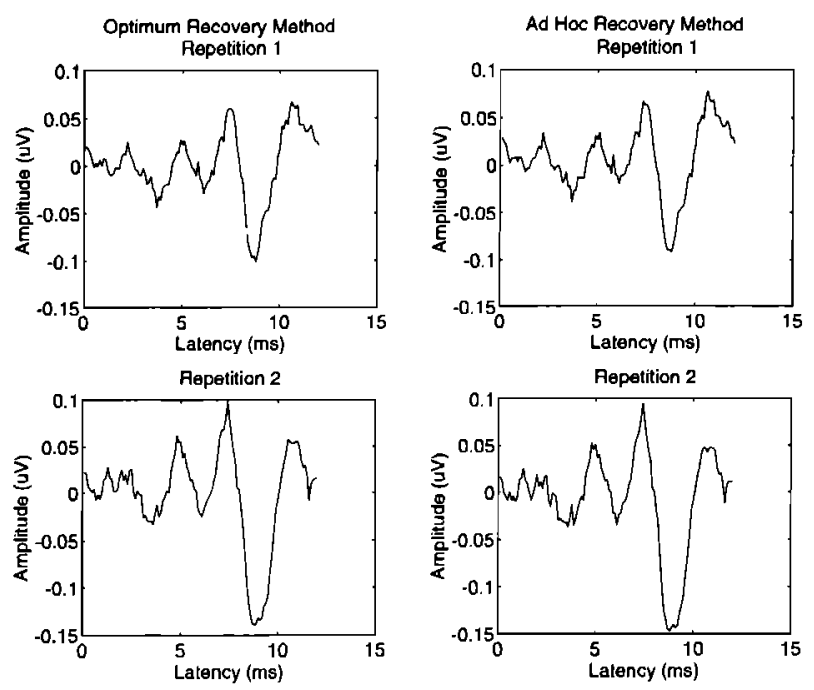

FIG. 6. Recovered ABRs using optimal white noise and conventional recovery methods. The stimulus sequence was an order of 6 MLS with MPI $=2.2$ ms presented at $90 \mathrm{~dB}$ SPL. Each repetition is the average of 200 responses.

tively modest (at maximum, a factor of 2) and depends on a number of parameters. Such an endeavor is beyond the scope of the present study, which is to provide a general framework for characterizing the recovery of responses to arbitrary stimulus sequences.

\section{DISCUSSION}

In an effort to consider the data collection time implications of cross-correlation techniques for recording evoked responses, we develop a general framework to characterize the operations involved in recovering the responses of interest. This general framework is characterized by a matrixbased representation for the response to an arbitrary stimulus sequence. Presumably, the choice of the stimulus will be dictated by the purpose of the data collection. If the purpose is to study temporal nonlinearities the stimuli must include the range of temporal interactions of interest. A different sequence would be selected if amplitude or frequency nonlinearities were of interest.

This framework employs linear algebraic techniques to analyze the recovery process. There are, in general, an infinite number of recovery sequences that can "recover" the response of the system to the stimuli constituting the stimulation sequence. Again, the selection of the most suitable recovery sequence will depend upon the demands of the recording session. We develop an expression for the recovery sequence that minimizes the noise variance in the response and therefore maximizes the SNR. For other applications the most computationally efficient recovery sequence might be preferred although systematic means for determining such recovery sequences do not exist.

It is clear that the recovery sequence affects the noise. This has not been generally recognized, and, therefore, deserves attention. First, the recovery sequence colors the noise. This is true even if the background noise during data collection is perfectly white. Second, the variance of the noise may vary as a function of time, i.e., the noise variance may not be the same at all points along the response waveform. Third, the recovery sequence that minimizes the noise variance can be identified. This recovery sequence is the most efficient in terms of improving the SNR in a given amount of time.

We use this general framework to evaluate the relative merits of conventional and MLS evoked response recordings as applied to ABRs, the most frequent EP application of MLS techniques. We show that with white background noise relative efficiency is dependent upon the ratio of response length to MPI and the amplitude loss as a function of stimulus rate. This analysis assumes that the conventional responses are recorded at the maximum possible rate. Using measured amplitude loss rate functions, we show that conventional averaging is two to five times as efficient as the MLS method depending on the MPI and recovery sequence. Although taking different approaches, other researchers have reached similar conclusions (Burkard, 1991; Chan et al., 1992; Marsh, 1992; Picton et al., 1992; Lasky et al., 1993). The generality of our approach is further illustrated by an example that compares efficiencies for colored noise with a dominant low-frequency component.

Several comments are in order. First, artifact rejection has not been considered in our comparison. Second, it is clear that more and better estimates of the ABR amplitude reduction associated with increasing stimulus rates are needed in order to better compare the relative speed of MLS and conventional signal averaging. Third, ABR amplitude $X$ rate functions vary from subject to subject and, consequently, conclusions depend upon the subjects tested. In particular, newborns seem to have shallower functions than adults (Lasky et al., 1992). Consequently, MLS recordings should be relatively more efficient in newborns than adults. Fourth, at lower intensities ABR amplitude reduction as a function of rate is less than at higher intensities (Burkard, 1991; Lasky et al., 1993). This would make MLS recordings relatively more efficient at low intensities. Fifth, the duration of the ABR is in question. For example, Picton et al. (1992) define ABR duration to be $15 \mathrm{~ms}$ in adults, presumably longer in newborns. The longer the response length the greater the relative efficiency of MLS recordings. Sixth, the example we selected concerned ABRs. Different conclusions may well apply to other evoked responses. Indeed, Picton et al. (1992) indicated that time savings in data collection might be realized with MLS middle latency auditory-evoked responses but doubted much time savings with MLS ABRs or late auditory-evoked responses. Seventh, MLS techniques can be used to present simultaneously multiple stimulus sequences and recover separate responses to each of those sequences. Sutter and Tran (1991) have used this capability to simultaneously record over 241 local electro-retinograms, a task that could not be accomplished in a feasible amount of time using conventional techniques. Lasky et al. (1993) have recorded simultaneous bilateral ABR recordings which required roughly half the data collection time as separate and sequential monaural ABR MLS recordings. Finally, even if these sequences offer no recording time savings, there are other reasons for using them. Most importantly, they can be used to investigate nonlinearities evoked by the interactions 
among the stimuli constituting the stimulating sequences (Sutter, 1987; Sutter and Vaegan, 1990; Ying and Hecox, 1991; Lasky et al., 1992). Furthermore, they can be used to investigate responses that superimpose to define "steadystate" evoked responses (Picton et al., 1992).

\section{ACKNOWLEDGMENT}

This work was supported in part by the National Institutes of Health NINCDS Grant No. NSR 0116436.

\section{APPENDIX: SOLUTION TO (9) USING THE METHODS OF LAGRANGE MULTIPLIERS AND COMPLETING THE SQUARE}

Here we obtain the solution to (9) using the methods of Lagrange multipliers and completing the square. Let $2 \mathbf{L}$ be an $M$ by $M$ matrix of Lagrange multipliers. The cost function in (9) is augmented with the inner product of the Lagrange multipliers and the constraint to obtain the Lagrangian $L(\mathbf{R}, \mathbf{L})$ :

$$
L(\mathbf{R}, \mathbf{L})=\operatorname{tr}\left[\mathbf{R S R}^{T}+(\mathbf{R A}-\mathbf{I}) 2 \mathbf{L}\right]
$$

Noting that $\operatorname{tr} B=\operatorname{tr} B^{T}$, we rewrite (A1) as

$$
L(\mathbf{R}, \mathbf{L})=\operatorname{tr}\left[\mathbf{R S R}^{T}+(\mathbf{R A}-\mathbf{I}) \mathbf{L}+\mathbf{L}^{T}\left(\mathbf{A}^{T} \mathbf{R}^{T}-\mathbf{I}\right)\right] .
$$

It is easy to verify that (A2) can be expressed as the perfect square in $\mathbf{R}$ :

$$
\begin{aligned}
L(\mathbf{R}, \mathbf{L})= & \operatorname{tr}\left[\left(\mathbf{R}+\mathbf{L}^{T} \mathbf{A}^{T} \mathbf{S}^{-1}\right) \mathbf{S}\left(\mathbf{R}+\mathbf{L}^{T} \mathbf{A}^{T} \mathbf{S}^{-1}\right)^{T}-\mathbf{L}-\mathbf{L}^{T}\right. \\
& \left.-\mathbf{L}^{T} \mathbf{A}^{T} \mathbf{S}^{-1} \mathbf{A L}\right] .
\end{aligned}
$$

Only the first term in the brackets is a function of $\mathbf{R}$. The matrix $S$ is positive definite so the minimum of $L(R, L)$ is attained for

$$
\mathbf{R}=-\mathbf{L}^{T} \mathbf{A}^{T} \mathbf{S}^{-1} .
$$

The Lagrange multiplier matrix $L$ is now obtained by substituting $\mathbf{R}$ in the constraint $\mathbf{R A}=\mathbf{I}$ to obtain

$$
-\mathbf{L}^{T} \mathbf{A}^{T} \mathbf{S}{ }^{1} \mathbf{A}=\mathbf{I} \text {, }
$$

or

$$
\mathbf{L}^{T}=-\left(\mathbf{A}^{T} \mathbf{S}^{\mathbf{1}} \mathbf{A}\right)^{-1} .
$$

Substituting (A6) into (A4) we have the solution

$$
\mathbf{R}=\left(\mathbf{A}^{T} \mathbf{S}^{-1} \mathbf{A}\right)^{-1} \mathbf{A}^{T} \mathbf{S}^{-1}
$$

Berger, H. (1929). “Uber das elektrenkephalogramm des menschen," Arch. Psychiatr. Nervenkr. 87, 527-570.
Brewer, J. W. (1978). "Kronecker products and matrix calculus in system theory." IEEE Trans. Circuits Sysi. CAS-25, 772-781.

Burkard. R., Shi, Y., and Hecox, K. E. (1990a). "Brainstern auditory evoked responses elicited by maximum length sequences: Effects of simultaneous masking noise," J. Acoust. Soc. Am. 87, 1656-1664.

Burkard, R., Shi, Y., arid Hecox, K. E. (1990b). “A comparison of maximum length and Legendre sequences for the derivalion of BAERs at rapid rates of stimulation," J. Acoust. Soc. Am. 87, 1665-1672.

Burkard, R. (1991). "Hluman brain-stem auditory evoked responses obtained by cross correlation to trains of clicks, noise bursts, and tone bursts," J. Acoust. Soc. Am. 90, 1398-1404.

Chan, F., Lam, F., Poon, P., and Du, M. (1992). "Measurement of human BAERs by the maximum length sequence technique," Med. Biol. Eng. Computing 30, 32-40.

Davies, W. D. T. (1966). "Generation and properties of maximum length sequences," Control 10. 364-365.

Dawson, G. D. (1951). "A summation technique for delection of small amplitude signals in a large irregular background," J. Physiol. 115, 2-3.

Eysholdt, U., and Schreiner, C. (1982). "Maximum length sequences-A fast method for measuring brain-stem-evoked responses," Audiology 21, 242-250.

Hall, J. W. (1992). Handbook of Auditory Evoked Responses (Allyn and Bacon, Boston).

Kay, S. M. (1993). Fundamentals of Statistical Signal Processing: Estimation Theory (Prentice-Hall, Englewood Cliffs, NJ), pp. 133-156.

Lasky, R. E., Perlman, J., and Hecox, K. E. (1992). "Maximum length sequence auditory evoked brainstem responses in human newborns and adults," J. Am. Acad. Audiol. 3, 383-389.

Lasky, R. E., Shi, Y., and Hecox, K. E. (1993). "Binaural maximum length sequence auditory cvoked brainstem responses in human adults," J. Acoust. Soc. Am. 93, 2077-2087.

Lasky, R. E., and Rupert, A. (1982). “Temporal masking of auditory evoked brainstem responses in human newborns and adults," Hear. Res. 6, 315334.

Marsh, R. (1992). "Signal-to-noise constrainls on maximum-lengthsequence auditory brain stem responses," Ear Hear. 13, 396-400.

Muirhead, R. J. (1982). Aspects of Multivariate Statistical Theory (Wiley, New York), p. 82.

Noble, B., and Daniel, J. W. (1977). Applied Linear Algebra, 2nd ed. (Prentice-Hall, Englewood Clifts, NJ).

Picton, T., Champagne. S. C., and Kellet1, A. J. C. (1992). "Human auditory evoked potentials recorded using maximum length sequences," Electroencephalogr. Clin. Neurophysiol. 84, 90-100.

Sutter, E. E. (1987). "A practical nonstochastic approach to nonlinear timedomain analysis," in Proceedings, Biomedical Simulations Resource, Advanced Methods of I'hysiological System Modeling, edited by V. Z. Marmarelis (Biomedical Simulations Resource, University of Southern California, Lus Angeles), Vol. 1, pp. 303-315.

Sutter, E. E., and Tran, D. (1991). "Identitication of pattern ERG components by means of the ir ficld topography," The Association for Research in Vision and Opthamology. Annual Spring Meeting, Sarasota, FL, April 28May 3; abstract \#1283, p. 929.

Sutter, E. E., and Vaegen (1990). "Lateral interaction component and local luminance nonlinearities in human pattern ERG," Vision Res. 30, 659671.

Ying, S., and Hecox. K. (1991). "Nonlinear system identification by $M$-pulse sequences: Application to brainstem auditory evoked responses," IEEE Trans. Biomed. Eng. 38, 834-845. 